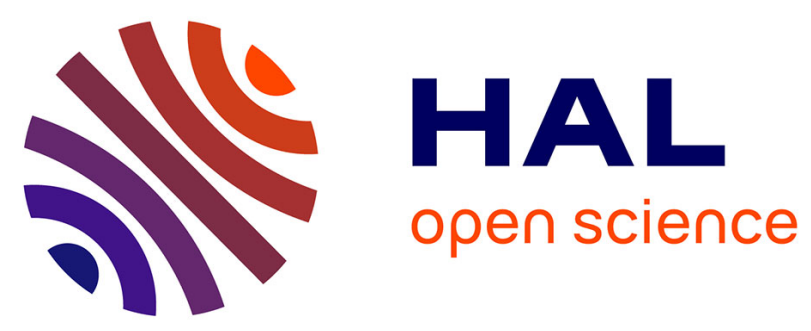

\title{
ON CERTAIN TANNAKIAN CATEGORIES OF INTEGRABLE CONNECTIONS OVER KÄHLER MANIFOLDS
}

Indranil Biswas, Joao Pedro dos Santos, Sebastian Heller, Sorin Dumitrescu

\section{- To cite this version:}

Indranil Biswas, Joao Pedro dos Santos, Sebastian Heller, Sorin Dumitrescu. ON CERTAIN TANNAKIAN CATEGORIES OF INTEGRABLE CONNECTIONS OVER KÄHLER MANIFOLDS. Canadian Journal of Mathematics = Journal Canadien de Mathématiques, In press. hal-02923470

\author{
HAL Id: hal-02923470 \\ https://hal.science/hal-02923470
}

Submitted on 27 Aug 2020

HAL is a multi-disciplinary open access archive for the deposit and dissemination of scientific research documents, whether they are published or not. The documents may come from teaching and research institutions in France or abroad, or from public or private research centers.
L'archive ouverte pluridisciplinaire HAL, est destinée au dépôt et à la diffusion de documents scientifiques de niveau recherche, publiés ou non, émanant des établissements d'enseignement et de recherche français ou étrangers, des laboratoires publics ou privés. 


\title{
ON CERTAIN TANNAKIAN CATEGORIES OF INTEGRABLE CONNECTIONS OVER KÄHLER MANIFOLDS
}

\author{
INDRANIL BISWAS, JOÃO PEDRO DOS SANTOS, SORIN DUMITRESCU, \\ AND SEBASTIAN HELLER
}

\begin{abstract}
Given a compact Kähler manifold $X$, it is shown that pairs of the form $(E, D)$, where $E$ is a trivial holomorphic vector bundle on $X$, and $D$ is an integrable holomorphic connection on $E$, produce a neutral Tannakian category. The corresponding pro-algebraic affine group scheme is studied. In particular, it is shown that this proalgebraic affine group scheme for a compact Riemann surface determines uniquely the isomorphism class of the Riemann surface.
\end{abstract}

\section{INTRODUCTION}

A question of Ghys asks the following: Is there a pair of the form $(M, D)$, where $M$ is a compact Riemann surface of genus at least two, and $D$ is an irreducible holomorphic $\mathrm{SL}(2, \mathbb{C})$-connection on the rank two trivial holomorphic vector bundle $\mathcal{O}_{M}^{\oplus 2}$, such that the image of the monodromy homomorphism for $D$ is contained in a cocompact lattice of $\mathrm{SL}(2, \mathbb{C})$. The motivation for this question comes from the study of compact quotients of $\mathrm{SL}(2, \mathbb{C})$ by lattices. Such quotients are compact non-Kähler manifolds. While they can't contain a complex surface [HM, p. 239, Theorem 2], it is not known whether they can contain compact Riemann surfaces of genus $g>1$. A positive answer to Ghys' question would provide a nontrivial holomorphic map from the Riemann surface $M$ to the quotient of $\mathrm{SL}(2, \mathbb{C})$ by the cocompact lattice containing the image of the monodromy homomorphism for $D$. In fact the two problems are equivalent (see [CDHL] for explanations for the origin of Ghys' question).

Let us also mention that a related question of characterizing rank two holomorphic vector bundles $\mathcal{V}$ over a compact Riemann surface, such that for some holomorphic connection on $\mathcal{V}$ the image of the associated monodromy homomorphism is Fuchsian, was raised in [Ka, p. 556] (there this question is attributed to Bers).

The above, still open questions of Bers and Ghys, and some related questions in [CDHL, Ka], motivated us to investigate the holomorphic connections on a trivial holomorphic vector bundle. Answering a question asked in [CDHL], examples of irreducible holomorphic $\mathrm{SL}(2, \mathbb{C})$-connection with real monodromy, on the trivial holomorphic $\mathrm{SL}(2, \mathbb{C})$ bundle over a compact Riemann surface, were constructed in [BDH].

2010 Mathematics Subject Classification. 53C07, 14C34, 16D90, $14 \mathrm{~K} 20$.

Key words and phrases. Integrable holomorphic connection, Higgs bundle, neutral Tannakian category, complex torus, Torelli theorem. 
Here we consider integrable holomorphic connections on trivial holomorphic vector bundles over a compact Kähler manifold $X$. Once we fix a base point $x_{0} \in X$ in order to define a fiber functor, using the Tannakian category theory it is shown that this category produces a quotient of the pro-algebraic completion $\varpi\left(X, x_{0}\right)$ of the fundamental group $\pi_{1}\left(X, x_{0}\right)$ (the details are in Section 3); this quotient is denoted by $\Theta\left(X, x_{0}\right)$. Then we prove a Torelli type Theorem with respect to $\Theta\left(X, x_{0}\right)$, for compact Riemann surfaces and also for compact complex tori.

The main results of Section 3 and Section 4 are the following:

(1) For compact Kähler manifolds $X$ and $Y$, the natural homomorphism

$$
\Theta\left(X, x_{0}\right) \times \Theta\left(Y, y_{0}\right) \longrightarrow \Theta\left(X \times Y,\left(x_{0}, y_{0}\right)\right)
$$

is an isomorphism. (See Proposition 3.9.)

(2) Let $\beta: X \longrightarrow Y$ be an orientation preserving diffeomorphism between compact Riemann surfaces such that the corresponding homomorphism

$$
\beta_{\natural}: \varpi\left(X, x_{0}\right) \longrightarrow \varpi\left(Y, \beta\left(x_{0}\right)\right)
$$

descends to a homomorphism from $\Theta\left(X, x_{0}\right)$ to $\Theta\left(Y, \beta\left(x_{0}\right)\right)$. Then the two Riemann surfaces $X$ and $Y$ are isomorphic. (See Theorem 4.1.)

(3) Let $\varphi: \mathbb{T} \longrightarrow \mathbb{S}$ be a diffeomorphism between two compact complex tori such that the corresponding homomorphism

$$
\varphi_{*}: \varpi\left(\mathbb{T}, x_{0}\right) \longrightarrow \varpi\left(\mathbb{S}, \varphi\left(x_{0}\right)\right)
$$

descends to a homomorphism from $\Theta\left(\mathbb{T}, x_{0}\right)$ to $\Theta\left(\mathbb{S}, \varphi\left(x_{0}\right)\right)$. Then there is a biholomorphism

$$
\mathbb{T} \longrightarrow \mathbb{S}
$$

which is homotopic to the map $\varphi$. (See Proposition 4.2.)

In Section 5 we consider integrable holomorphic connections on holomorphic vector bundles over $X$ which decompose into a direct sum of holomorphic line bundles. Using the Tannakian category theory in a similar way, we show that this category also produces a quotient $\Delta\left(X, x_{0}\right)$ of the pro-algebraic completion $\varpi\left(X, x_{0}\right)$ of the fundamental group $\pi_{1}\left(X, x_{0}\right)$. Then we adapt our methods in Section 4 in order to prove the same Torelli type theorems for $\Delta\left(X, x_{0}\right)$.

As is well-known, the category of vector bundles easily fails to be abelian, but as observed as far back as [Se, Propsoition 3.1], semi-stability can be brought in to mend this failure and produce interesting abelian categories of vector bundles. Section 6 connects the group scheme $\Theta$ to such an idea. Using the abelian category of pseudostable vector bundles to produce an affine group scheme $\pi^{\mathrm{S}}\left(X, x_{0}\right)$, we show that $\Theta\left(X, x_{0}\right) \times \pi^{\mathrm{S}}\left(X, x_{0}\right)$ captures a large piece of $\varpi\left(X, x_{0}\right)$; see Theorem 6.5. The construction of $\pi^{\mathrm{S}}\left(X, x_{0}\right)$ crucially uses foundational works of Simpson. 


\section{Neutral TANnAKian CATEgories FOR a KÄHLER MANifold}

Let $X$ be a compact connected Kähler manifold of complex dimension $d$. Fix a Kähler form $\omega$ on $X$. The degree of a torsionfree coherent analytic sheaf $F$ on $X$ is defined to be

$$
\operatorname{degree}(F):=\left(c_{1}(\operatorname{det} F) \cup \omega^{d-1}\right) \cap[X] \in \mathbb{R} .
$$

Here and elsewhere, we write $c_{i}$ for the $i$-th Chern class in $H_{d R}^{2 i}(X, \mathbb{R})$ and $\operatorname{define} \operatorname{det} F$ following $[\mathrm{Ko}, \mathrm{Ch} . \mathrm{V}, \S 6]$. The real number

$$
\mu(F):=\frac{\operatorname{degree}(F)}{\operatorname{rank}(F)}
$$

is called the slope of $F$.

Fix a base point $x_{0} \in X$. Let

$$
\phi: \pi_{1}\left(X, x_{0}\right) \longrightarrow \varpi\left(X, x_{0}\right)
$$

be the pro-algebraic completion of the fundamental group $\pi_{1}\left(X, x_{0}\right)$. We recall that $\varpi\left(X, x_{0}\right)$ is a pro-algebraic affine group scheme over $\mathbb{C}$ which is uniquely characterized by the following property: for any homomorphism

$$
\gamma: \pi_{1}\left(X, x_{0}\right) \longrightarrow G
$$

to a complex affine algebraic group $G$, there is a unique algebraic homomorphism

$$
\widehat{\gamma}: \varpi\left(X, x_{0}\right) \longrightarrow G
$$

such that $\widehat{\gamma} \circ \phi=\gamma$. There are mainly two equivalent constructions of $\varpi\left(X, x_{0}\right)$ : one by means of the Tannakian category of finite dimensional representations of $\pi_{1}\left(X, x_{0}\right)$ and Tannakian duality [DMOS] and the other by Freyd's adjoint functor theorem applied to the $\mathbb{C}$-points functor from group schemes to groups [Fr, p. 84, 3.J].

We shall recall from $[\mathrm{Si} 2$, p. 70$]$ three neutral Tannakian categories associated to the pointed Kähler manifold $\left(X, x_{0}\right)$ which, in particular, furnish a Tannakian description of $\varpi\left(X, x_{0}\right)$.

Let $\mathcal{C}_{d R}(X)$ denote the category whose objects are pairs of the form $(E, D)$, where $E$ is a holomorphic vector bundle on $X$ and $D$ is an integrable holomorphic connection on $E$ (see [At] for holomorphic connections). Morphisms from $(E, D)$ to $\left(E^{\prime}, D^{\prime}\right)$ are all holomorphic homomorphisms of vector bundles $h: E \longrightarrow E^{\prime}$ that intertwine the connections $D$ and $D^{\prime}$, meaning $D^{\prime} \circ h=\left(h \otimes \operatorname{Id}_{\Omega_{X}^{1}}\right) \circ D$ as differential operators from $E$ to $E^{\prime} \otimes \Omega_{X}^{1}$ with $\Omega_{X}^{1}$ being the holomorphic cotangent bundle of $X$. This category is equipped with the operators of direct sum, tensor product and dualization. More precisely, $\mathcal{C}_{d R}(X)$ is a rigid abelian tensor category (see [DMOS, p. 118, definition 1.14] for rigid abelian tensor categories). It is straightforward to check that this category $\mathcal{C}_{d R}(X)$, equipped with the faithful fiber functor that sends any object $(E, D)$ to the fiber $\left.E\right|_{x_{0}}$ over $x_{0} \in X$, defines a neutral Tannakian category (see [DMOS, p. 138, Definition 2.19], [Sa], [Si2, p. 67], [No, p. 76] for neutral Tannakian category). Given any neutral Tannakian category, a theorem of Saavedra Rivano associates to it a pro-algebraic affine group scheme over $\mathbb{C}$ [DMOS, p. 130, Theorem 2.11] (and the remark following [DMOS, p. 138, Definition 2.19]), [Sa], 
[No, p. 77, Theorem 1.1], [Si2, p. 69]. Therefore, the neutral Tannakian category $\mathcal{C}_{d R}(X)$ corresponds to a pro-algebraic affine group scheme over $\mathbb{C}$.

Let $\mathcal{C}_{B}(X)$ denote the category whose objects are all finite dimensional complex representations of $\pi_{1}\left(X, x_{0}\right)$. Using the tautological fiber functor, it defines a neutral Tannakian category. The pro-algebraic affine group scheme over $\mathbb{C}$ corresponding to $\mathcal{C}_{B}(X)$, by the above mentioned theorem of Saavedra Rivano ([Sa], [DMOS, p. 130, Theorem 2.11]), is $\varpi\left(X, x_{0}\right)$ in $(2.1)$ [Si2, p. 69, Lemma 6.1].

A Higgs bundle on $X$ is a pair of the form $(E, \theta)$, where $E$ is a holomorphic vector bundle of $X$ and $\theta \in H^{0}\left(X, \operatorname{End}(E) \otimes \Omega_{X}^{1}\right)$ with $\theta \wedge \theta=0$ [Si1], [Si2]; the holomorphic section $\theta$ is called a Higgs field on $E$. A Higgs bundle $(E, \theta)$ is called stable (respectively, semistable) if

$$
\mu(F)<\mu(E) \quad \text { (respectively, } \mu(F) \leq \mu(E) \text { ) }
$$

for every coherent analytic subsheaf $F \subset E$ with $0<\operatorname{rank}(F)<\operatorname{rank}(E)$ and $\theta(F) \subset$ $F \otimes \Omega_{X}^{1}$.

Let $\mathcal{C}_{D o l}(X)$ denote the category whose objects are Higgs bundles $(E, \theta)$ such that $c h_{2}(E) \cup \omega^{d-2}=0$ and there is a filtration of holomorphic subbundles

$$
0=E_{0} \subset E_{1} \subset \cdots \subset E_{i} \subset \cdots \subset E_{\ell-1} \subset E_{\ell}=E
$$

satisfying the following three conditions:

(1) $\theta\left(E_{j}\right) \subset E_{j} \otimes \Omega_{X}^{1}$ for all $1 \leq j \leq \ell$,

(2) degree $\left(E_{j} / E_{j-1}\right)=0$ for all $1 \leq j \leq \ell$, and

(3) the Higgs bundle $\left(E_{j} / E_{j-1}, \theta\right)$ is stable for all $1 \leq j \leq \ell$ (the Higgs field on $E_{j} / E_{j-1}$ induced by $\theta$ is also denoted by $\theta$ ).

In $[\mathrm{BG}]$ such Higgs bundles are called pseudostable.

A homomorphism from $(E, \theta)$ to $\left(E^{\prime}, \theta^{\prime}\right)$ is a holomorphic homomorphism

$$
h: E \longrightarrow E^{\prime}
$$

such that $\theta^{\prime} \circ h=\left(h \otimes \operatorname{Id}_{\Omega_{X}^{1}}\right) \circ \theta$ as homomorphisms from $E$ to $E^{\prime} \otimes \Omega_{X}^{1}$. It is known that $\mathcal{C}_{D o l}(X)$ is a rigid abelian tensor category [Si2, p. 70]. This category $\mathcal{C}_{D o l}(X)$ admits the faithful fiber functor that sends any object $(E, \theta)$ to the fiber $\left.E\right|_{x_{0}}$. In other words, $\mathcal{C}_{\text {Dol }}(X)$ is a neutral Tannakian category.

The two categories $\mathcal{C}_{d R}(X)$ and $\mathcal{C}_{B}(X)$ are equivalent by the Riemann-Hilbert correspondence that assigns to a flat connection the corresponding monodromy representation. Using fundamental theorems of Corlette, [Co], and Simpson, [Si1], in [Si2] Simpson proved that the category $\mathcal{C}_{D o l}(X)$ is equivalent to $\mathcal{C}_{d R}(X)$ (see [Si2, p. 36, Lemma 3.5], $\left[\mathrm{Si} 2\right.$, p. 70]). Therefore, all these three neutral Tannakian categories, namely $\mathcal{C}_{d R}(X)$, $\mathcal{C}_{B}(X)$ and $\mathcal{C}_{D o l}(X)$, produce the same pro-algebraic affine group scheme over $\mathbb{C}$ using the theorem of Saavedra Rivano mentioned earlier ([Sa], [DMOS, p. 130, Theorem 2.11]). In other words, each of these three neutral Tannakian categories produces the pro-algebraic affine group scheme $\varpi\left(X, x_{0}\right)$ in $(2.1)$. 
When $X$ is a smooth complex projective variety, and the cohomology class of the closed form $\omega$ is rational, then the category $\mathcal{C}_{D o l}(X)$ coincides with the category of semistable Higgs bundles $(E, \theta)$ on $X$ with $c h_{2}(E) \cup \omega^{d-2}=0$ and degree $(E)=0$ [Si2, p. 39, Theorem 2].

\section{The Tannakian subcategory $\mathcal{T}_{d R}$ OF $\mathcal{C}_{d R}$}

\subsection{The Tannakian category and the associated group scheme.}

Definition 3.1. Let $\mathcal{T}_{d R}(X)$ be the full subcategory of $\mathcal{C}_{d R}(X)$ (defined in Section 2) whose objects are all the couples $(E, D)$ satisfying the condition that the holomorphic vector bundle $E$ is holomorphically trivial.

Clearly, $\mathcal{T}_{d R}(X)$ is stable under tensor products and duals. The identity object $\left(\mathcal{O}_{X}, \mathrm{~d}\right)$, where $\mathrm{d}$ is the de Rham differential, certainly is an object of $\mathcal{T}_{d R}(X)$. In addition $\mathcal{T}_{d R}(X)$ is stable under quotients, as shown by the next proposition.

Proposition 3.2. Let $(E, D)=\left(\mathcal{O}_{X}^{\oplus r}, D\right)$ be an object of $\mathcal{T}_{d R}(X)$ and

$$
q:\left(\mathcal{O}_{X}^{\oplus r}, D\right) \longrightarrow\left(E^{\prime}, D^{\prime}\right)
$$

an epimorphism in $\mathcal{C}_{d R}(X)$. Then the holomorphic vector bundle $E^{\prime}$ is also trivial.

Proof. We note that degree $\left(E^{\prime}\right)=0$ because $E^{\prime}$ carries an integrable connection [At, pp. 192-193, Theorem 4], [GH, p. 141, Proposition]. Let $r^{\prime}$ be the rank of $E^{\prime}$, and introduce $\operatorname{Gr}\left(r, r^{\prime}\right)$, the Grassmann manifold of $r^{\prime}$ dimensional quotients of $\mathbb{C}^{r}$. Note that the trivial bundle $\mathcal{O}_{\mathrm{Gr}\left(r, r^{\prime}\right)}^{\oplus r}$ comes with a tautological quotient of rank $r^{\prime}$, call it $\mathcal{O}_{\mathrm{Gr}\left(r, r^{\prime}\right)}^{\oplus r} \longrightarrow U$. Then, $q$ induces a morphism $f: X \longrightarrow \operatorname{Gr}\left(r, r^{\prime}\right)$ such that $E^{\prime}=f^{*} U$. In particular, we have $c_{1}\left(f^{*}(\operatorname{det} U)\right)=c_{1}\left(E^{\prime}\right)=0$. The fact that $\operatorname{det}(U)$ is ample implies that $f$ is a constant map [GH, p. 177]. Hence, $E^{\prime}$ is holomorphically trivial.

Corollary 3.3. The full subcategory $\mathcal{T}_{d R}(X)$ of $\mathcal{C}_{d R}(X)$ is an abelian subcategory.

Proof. Let $\alpha:(E, D) \longrightarrow\left(E^{\prime}, D^{\prime}\right)$ be an arrow in $\mathcal{T}_{d R}(X)$. Now, Image $(\alpha)$ is a subconnection, call it $\left(I, D^{\prime}\right)$, of $\left(E^{\prime}, D^{\prime}\right)$ and we have an epimorphism $\alpha:(E, D) \longrightarrow$ $\left(I, D^{\prime}\right)$ in $\mathcal{C}_{d R}(X)$. We conclude from Proposition 3.2 that $\left(I, D^{\prime}\right)$ is an object of $\mathcal{T}_{d R}(X)$. Again from Proposition 3.2 it follows that $\operatorname{coker}(\alpha)$ is an object of $\mathcal{T}_{d R}(X)$ because it is a quotient of an object of $\mathcal{T}_{d R}(X)$. Finally, working with duals, these arguments give that $\operatorname{kernel}(\alpha)$ is also in $\mathcal{T}_{d R}(X)$. Hence, the standard criterion for a subcategory to be an abelian subcategory can be applied [Fr, Theorem 3.41].

Let

$$
\Theta\left(X, x_{0}\right)
$$

be the affine group scheme over $\mathbb{C}$ corresponding to $\mathcal{T}_{d R}(X)$ via [DMOS, p. 130, Theorem 2.11] by means of the exact functor $\left.(E, D) \longmapsto E\right|_{x_{0}}$. From Proposition 3.2 and the 
standard criterion [DMOS, Proposition 2.21, p.139] we conclude that the natural arrow of group schemes

$$
\mathbf{q}_{X}: \varpi\left(X, x_{0}\right) \longrightarrow \Theta\left(X, x_{0}\right)
$$

is a quotient homomorphism.

Clearly, for any given holomorphic map $f: Y \longrightarrow X$, we obtain tensor functors

$$
f^{\#}: \mathcal{C}_{d R}(X) \longrightarrow \mathcal{C}_{d R}(Y)
$$

and

$$
f^{\#}: \mathcal{T}_{d R}(X) \longrightarrow \mathcal{T}_{d R}(Y)
$$

defined by $(E, D) \longmapsto\left(f^{*} E, f^{*} D\right)$. If, in addition, we let $y_{0} \in Y$ be a point which is taken to $x_{0}$, we derive homomorphisms of group schemes

$$
f_{\natural}: \varpi\left(Y, y_{0}\right) \longrightarrow \varpi\left(X, x_{0}\right)
$$

and

$$
f_{\natural}: \Theta\left(Y, y_{0}\right) \longrightarrow \Theta\left(X, x_{0}\right) .
$$

Using the equivalence of categories between $\mathcal{C}_{D o l}(X)$ and $\mathcal{C}_{d R}(X)$ mentioned in Section 2 , the above subcategory $\mathcal{T}_{d R}(X)$ of $\mathcal{C}_{d R}(X)$ gives a Tannakian full subcategory of $\mathcal{C}_{D o l}(X)$. It is natural to ask the following:

Question 3.4. What is a direct description of the Tannakian full subcategory of $\mathcal{C}_{D o l}(X)$ corresponding to the subcategory $\mathcal{T}_{d R}(X)$ of $\mathcal{C}_{d R}(X)$ ?

3.2. Characters of $\Theta$ : multiplicative and additive. Let $\mathbb{G}_{m}$ be the multiplicative group of nonzero scalars and $\mathbb{G}_{a}$ the additive group of scalars. Let $G$ be an affine group scheme over $\mathbb{C}$. Two of the most basic abstract groups associated to it are its group of characters $\operatorname{Hom}\left(G, \mathbb{G}_{m}\right)$ and its group of additive characters $\operatorname{Hom}\left(G, \mathbb{G}_{a}\right)$ (notations are those of [Wa, p. $5,1.2])$. These are denoted respectively by $\mathbb{X}(G)$ and $\mathbb{X}_{a}(G)$ in what follows. (We note that $\mathbb{X}_{a}(G)$ comes with the extra structure of a $\mathbb{C}$-vector space.) Clearly, $\mathbb{X}(G)$ is the group of isomorphism classes of rank one representations of $G$. In turn, the standard immersion of $\mathbb{G}_{a}$ into $\mathrm{GL}(2, \mathbb{C})$ permits us to view $\mathbb{X}_{a}(G)$ as the vector space $\operatorname{Ext}_{G}(\mathbf{1}, \mathbf{1})$ parametrizing extensions of the trivial representation by itself. We shall record some observations on characters and additive characters of $\varpi\left(X, x_{0}\right)$ and $\Theta\left(X, x_{0}\right)$. In what follows,

$$
\operatorname{Pic}^{\tau}(X)
$$

is the subgroup of $\operatorname{Pic}(X)$ formed by the classes of line bundles with vanishing first Chern class. Recall that according to our convention, Chern classes are real. The subgroup $\operatorname{Pic}^{0}(X)$ of classes having vanishing integral first Chern class is of finite index in $\operatorname{Pic}^{\tau}(X)$ as we learn from the theorem of the base.

Lemma 3.5. The map

$$
H^{0}\left(X, \Omega_{X}^{1}\right) \longrightarrow \mathbb{X}\left(\Theta\left(X, x_{0}\right)\right)
$$


sending a form $\alpha$ to the isomorphism class $\left(\mathcal{O}_{X}, \mathrm{~d}+\alpha\right)$ is an isomorphism. The group $\mathbb{X}\left(\varpi\left(X, x_{0}\right)\right)$ sits in a short exact sequence

$$
1 \longrightarrow \mathbb{X}\left(\Theta\left(X, x_{0}\right)\right) \longrightarrow \mathbb{X}\left(\varpi\left(X, x_{0}\right)\right) \longrightarrow \operatorname{Pic}^{\tau}(X) \longrightarrow 1
$$

Proof. The first claim is very simple and its verification is omitted. Then, if $(L, \nabla)$ is an integrable connection with $L$ a holomorphic line bundle, the interpretation of $c_{1}(L)$ as curvature form [GH, p. 141, Proposition] assures that $L \in \operatorname{Pic}^{\tau}(X)$, and we obtain the arrow $\mathbb{X}\left(\varpi\left(X, x_{0}\right)\right) \longrightarrow \operatorname{Pic}^{\tau}(X)$. The kernel is precisely the group of (isomorphism classes of $)$ objects in $\mathcal{T}_{d R}(X)$ of rank one, which is $\mathbb{X}\left(\Theta\left(X, x_{0}\right)\right)$. That any class in $\operatorname{Pic}^{\tau}(X)$ carries an integrable connection is explained by [LT, p. 40, Corollary 1.3.12].

We shall now be concerned with additive characters or, with the vector spaces

$$
\mathbb{X}_{a}\left(\varpi\left(X, x_{0}\right)\right) \simeq \operatorname{Ext}_{\mathcal{C}_{d R}(X)}(\mathbf{1}, \mathbf{1})
$$

and

$$
\mathbb{X}_{a}\left(\Theta\left(X, x_{0}\right)\right) \simeq \operatorname{Ext}_{\mathcal{T}_{d R}(X)}(\mathbf{1}, \mathbf{1}),
$$

where $\mathbf{1}$ is the identity object of the pertinent categories.

Let $H_{d R}^{1}(X, \mathbb{C})$ be $\mathbb{H}^{1}\left(X, \Omega_{X}^{\bullet}\right)[\mathrm{GH}$, p. 446], which is of course canonically isomorphic to $H^{1}(X, \mathbb{C})[\mathrm{GH}$, p. 448], and let

$$
\lambda_{X}: H^{0}\left(X, \Omega_{X}^{1}\right) \longrightarrow H_{d R}^{1}(X, \mathbb{C})
$$

be the natural arrow obtained from the fact that global holomorphic one forms on $X$ are closed.

Lemma 3.6. There are functorial isomorphisms of vector spaces

$$
\operatorname{Ext}_{\mathcal{C}_{d R}(X)}(\mathbf{1}, \mathbf{1}) \stackrel{\sim}{\longrightarrow} H_{d R}^{1}(X, \mathbb{C})
$$

and

$$
\operatorname{Ext}_{\mathcal{T}_{d R}(X)}(\mathbf{1}, \mathbf{1}) \stackrel{\sim}{\longrightarrow} H^{0}\left(X, \Omega_{X}^{1}\right) .
$$

Under these isomorphisms, the canonic arrow

$$
\operatorname{Ext}_{\mathcal{T}_{d R}(X)}(\mathbf{1}, \mathbf{1}) \longrightarrow \operatorname{Ext}_{\mathcal{C}_{d R}(X)}(\mathbf{1}, \mathbf{1})
$$

corresponds to $\lambda_{X}$ in (3.3).

Proof. Let $(E, \nabla) \in \mathcal{C}_{d R}(X)$ be an extension of $\left(\mathcal{O}_{X}, \mathrm{~d}\right)$ by itself. Let $\mathfrak{U}=\left\{U_{i}\right\}$ be an open covering of $X$ on which we have $e_{i} \in H^{0}\left(U_{i}, E\right)$ mapping to $1 \in \mathcal{O}_{X}\left(U_{i}\right)$. Consider the holomorphic 1-form $\theta_{i}$ on $U_{i}$ such that $\nabla\left(e_{i}\right)=1 \otimes \theta_{i}$. Then, on $U_{i} \cap U_{j}$, we have $e_{i}=a_{i j} \cdot 1+e_{j}$ with $a_{i j}$ a holomorphic function. The element

$$
\left(\theta_{i}, a_{i j}\right) \in C^{0}\left(\mathfrak{U}, \Omega_{X}^{1}\right) \oplus C^{1}\left(\mathfrak{U}, \mathcal{O}_{X}\right)
$$

defines a cocycle of the total Čech complex associated to the bicomplex $\left(C^{p}\left(\mathfrak{U}, \Omega_{X}^{q}\right)\right)_{0 \leq p, q}$ : the form $\theta_{i}$ is closed and $\mathrm{d} a_{i j}=\theta_{i}-\theta_{j}$. Hence, $\left\{\left(\theta_{i}, a_{i j}\right)\right\}$ gives an element of $H_{d R}^{1}(X, \mathbb{C})$, call it $[E, \nabla]$. Note that a 1-cocycle with coefficients in $\mathrm{GL}_{2}$ representing the vector bundle 
$E$ is given by the matrices $\left(\begin{array}{cc}1 & -a_{i j} \\ 0 & 1\end{array}\right)$ so that $E$ is isomorphic to $\mathcal{O}_{X}^{2}$ if and only if there exists, after eventually passing to a finer covering, a 0 -cochain $\left(h_{i}\right)$ with

$$
h_{j}-h_{i}=-a_{i j} .
$$

In this case, $[E, \nabla]$ belongs to the subspace $H^{0}\left(X, \Omega_{X}^{1}\right) \subset H_{d R}^{1}(X, \mathbb{C})$.

It is a lengthy but straightforward verification to show that

$$
\operatorname{Ext}_{\mathcal{C}_{d R}(X)}(\mathbf{1}, \mathbf{1}) \longrightarrow H_{d R}^{1}(X, \mathbb{C}),(E, \nabla) \longmapsto[E, \nabla]
$$

is bijective.

\subsection{Properties of the pro-algebraic group scheme $\Theta$.}

Lemma 3.7. Let $X$ and $Y$ be compact connected Kähler manifolds and $V$ a holomorphic vector bundle on $X \times Y$ of rank $r$ such that

- the restriction of $V$ to $\{x\} \times Y$ is holomorphically isomorphic to $\mathcal{O}_{Y}^{\oplus r}$ for every $x \in X$, and

- the restriction of $V$ to $X \times\{y\}$ is holomorphically isomorphic to $\mathcal{O}_{X}^{\oplus r}$ for every $y \in Y$.

Then $V$ is holomorphically isomorphic to $\mathcal{O}_{X \times Y}^{\oplus r}$.

Proof. Fix a point $y_{0} \in Y$. For any $x \in X$, the restriction $V_{x}$ of $V$ to $\{x\} \times Y$ trivializable. Hence the evaluation to $y_{0}$ of sections of $V_{x}$

$$
H^{0}\left(Y, V_{x}\right) \longrightarrow V_{x, y_{0}}, \sigma \longmapsto \sigma\left(y_{0}\right)
$$

is an isomorphism. This implies that for the natural projection $p^{X}: X \times Y \longrightarrow X$, the direct image of $V$ to $X$ is

$$
p_{*}^{X} V=V^{y_{0}}:=\left.V\right|_{X \times\left\{y_{0}\right\}} .
$$

The vector bundle $V^{y_{0}}$ is supposed to be holomorphically trivial. Fixing $r$ linearly independent holomorphic sections $\left\{s_{1}, \cdots, s_{r}\right\}$ of $V^{y_{0}}$, we get a holomorphic trivialization of $V^{y_{0}}$. Using the isomorphism in (3.4), each $s_{i}$ produces a section

$$
\widetilde{s}_{i} \in H^{0}\left(X, p_{*}^{X} V\right)=H^{0}(X \times Y, V) .
$$

Now these holomorphic sections $\left\{\widetilde{s}_{1}, \cdots, \widetilde{s}_{r}\right\}$ of $V$ trivialize $V$ holomorphically.

Proposition 3.8. The group scheme $\Theta\left(X, x_{0}\right)$ is the projective limit of connected algebraic groups.

Proof. Consider an algebraic quotient $\Theta\left(X, x_{0}\right) \longrightarrow \Theta^{\prime}$. We need to show that $\Theta^{\prime}$ is connected and this amounts to showing that if $\Theta^{\prime} \longrightarrow G$ is an algebraic quotient morphism to a finite group $G$, then $G$ is the trivial group. Now, write $R$ for the left regular representation of $G$ and note that the multiplication operation $R \otimes R \longrightarrow R$ and the identity $\mathbb{C} \longrightarrow R$ are $G$-equivariant. Transporting $R$ to the category $\mathcal{T}_{d R}(X)$ via the defining equivalence of $\Theta\left(X, x_{0}\right)$, we obtain an object $(\mathcal{R}, \nabla)$ of rank $|G|$ together with 
arrows $\mathcal{O}_{X} \longrightarrow \mathcal{R}$ and $\mathcal{R} \otimes \mathcal{R} \longrightarrow \mathcal{R}$ such that, endowed with them, $\mathcal{R}$ becomes a locally free $\mathcal{O}_{X}$-algebra. Note, in addition, that giving $\mathcal{R} \otimes \mathcal{R}$ the tensor product connection, the arrow $\mathcal{R} \otimes \mathcal{R} \longrightarrow \mathcal{R}$ is also horizontal.

Let $f: Y \longrightarrow X$ be the analytic spectrum of $\mathcal{R}$; it is a finite morphism of complex spaces [SC, Exposé 19] such that $f_{*} \mathcal{O}_{Y}=\mathcal{R}$. In addition, it is not hard to see that $f$ is a local biholomorphism so that $Y$ is a compact complex manifold. Indeed, we have a $G$-equivariant isomorphism of $R$-algebras $R \otimes R \longrightarrow R^{\oplus|G|}$ which allows us to see that for each $x \in X$, the $\mathcal{R}_{x}$-algebra $\mathcal{R}_{x} \otimes_{\mathcal{O}_{X, x}} \mathcal{R}_{x}$ (multiplication on the left) is isomorphic to $\mathcal{R}_{x}^{\oplus|G|}$. Consequently, $\mathcal{O}_{X, x} \longrightarrow \mathcal{R}_{x}$ is an étale morphism by flat descent.

Finally, $Y$ is connected since any idempotent $e \in H^{0}(X, \mathcal{R})$ is horizontal so that it produces a horizontal arrow $\mathcal{O}_{X} \longrightarrow \mathcal{R}$ and then a $G$-invariant element of $R$.

Because

$$
\begin{aligned}
1 & =\operatorname{dim} H^{0}\left(Y, \mathcal{O}_{Y}\right) \\
& =\operatorname{dim} H^{0}(X, \mathcal{R}) \\
& =|G| \cdot \operatorname{dim} H^{0}\left(X, \mathcal{O}_{X}\right),
\end{aligned}
$$

we conclude that $|G|=1$.

Proposition 3.9. Let $\left(X, x_{0}\right)$ and $\left(Y, y_{0}\right)$ be pointed compact Kähler manifolds. Set $P=X \times Y$, and let $i: X \longrightarrow P$ (respectively, $j: Y \longrightarrow P$ ) stand for the immersion defined by $x \longmapsto\left(x, y_{0}\right)$ (respectively, $y \longmapsto\left(x_{0}, y\right)$ ). Then, the arrow of affine group schemes

$$
\left(i_{\natural}, j_{\natural}\right): \Theta\left(X, x_{0}\right) \times \Theta\left(Y, y_{0}\right) \longrightarrow \Theta\left(P,\left(x_{0}, y_{0}\right)\right)
$$

is an isomorphism.

Proof. Let $p$ (respectively, $q$ ) be the natural projection of $P$ to $X$ (respectively, $Y$ ). In what follows, we abandon reference to the base points.

We know that the natural morphism

$$
\left(i_{\natural}, j_{\natural}\right): \varpi(X) \times \varpi(Y) \longrightarrow \varpi(P)
$$

is an isomorphism.

We claim that $i_{\natural}: \Theta(X) \longrightarrow \Theta(P)$ is a closed and normal immersion.

First note that $i_{\natural}$ is closed since $p_{\natural} \circ i_{\natural}: \Theta(X) \longrightarrow \Theta(X)$ is the identity map. Next, $i_{\natural}$ is normal since $i_{\natural}: \varpi(X) \longrightarrow \varpi(P)$ is a normal immersion and we posses the following commutative diagram

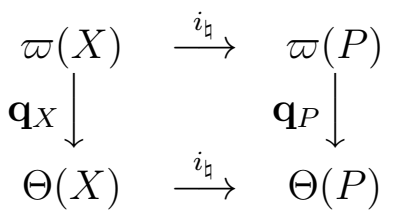

in which the vertical arrows are the quotient morphisms. This proves the claim.

Clearly, the same arguments apply to $j_{\natural}: \Theta(Y) \longrightarrow \Theta(P)$. 
Let

$$
\chi: \Theta(P) \longrightarrow Q
$$

be the cokernel of $i_{\natural}: \Theta(X) \longrightarrow \Theta(P)$ [Wa, 16.3, Theorem]. It is easy to see that the kernel of the composition

$$
\Theta(Y) \stackrel{j_{\natural}}{\longrightarrow} \Theta(P) \stackrel{\chi}{\longrightarrow} Q
$$

is trivial; indeed, $q_{\natural}: \Theta(P) \longrightarrow \Theta(Y)$ factors as

$$
\Theta(P) \stackrel{\chi}{\longrightarrow} Q \stackrel{r}{\longrightarrow} \Theta(Y)
$$

since $q_{\natural} \circ i_{\natural}$ is trivial, and $\operatorname{id}_{\Theta(Y)}=q_{\natural} \circ j_{\natural}$. We conclude that $\operatorname{Im}\left(i_{\natural}\right) \cap \operatorname{Im}\left(j_{\natural}\right)=\{e\}$. Now, a well-known lemma from group theory says that

$$
\left(i_{\natural}, j_{\natural}\right): \Theta(X) \times \Theta(Y) \longrightarrow \Theta(P)
$$

is a normal monomorphism of group schemes [Bo, I.4.9, p. 48, Proposition 15]. As such, it is a closed immersion [Wa, 15.3, Theorem]. Finally, since

$$
\left(i_{\natural}, j_{\natural}\right): \varpi(X) \times \varpi(Y) \longrightarrow \varpi(P)
$$

is a quotient morphism, we conclude that $\left(i_{\natural}, j_{\natural}\right): \Theta(X) \times \Theta(Y) \longrightarrow \Theta(P)$ is also one. Hence, $\Theta(X) \times \Theta(Y) \simeq \Theta(P)$.

Take a pointed compact Kähler manifold $\left(X, x_{0}\right)$. Let

$$
\Gamma \subset H^{0}\left(X, \Omega_{X}^{1}\right)^{*}
$$

be the image of the homomorphism $H_{1}(X, \mathbb{Z}) \longrightarrow H^{0}\left(X, \Omega_{X}^{1}\right)^{*}$ that sends any $\gamma \in$ $H_{1}(X, \mathbb{Z})$ to the element of the dual vector space $H^{0}\left(X, \Omega_{X}^{1}\right)^{*}$ defined by the integral

$$
\nu \longmapsto \int_{\gamma} \nu, \quad \nu \in H^{0}\left(X, \Omega_{X}^{1}\right)
$$

Let $\mathrm{A}(X)=H^{0}\left(X, \Omega_{X}^{1}\right)^{*} / \Gamma$ be the Albanese variety of $X$. Let

$$
\mathcal{A}: X \longrightarrow \mathrm{A}(X)
$$

be the Albanese map defined by

$$
\mathcal{A}(x)(\nu)=\int_{x_{0}}^{x} \nu
$$

this does not depend on the choice of path from $x_{0}$ to $x$. We have $H^{0}\left(X, \Omega_{X}^{1}\right)=$ $H^{0}\left(\mathrm{~A}(X), \Omega_{\mathrm{A}(X)}^{1}\right)$, and the homomorphism

$$
\alpha: H^{0}\left(\mathrm{~A}(X), \Omega_{\mathrm{A}(X)}^{1}\right) \longrightarrow H^{0}\left(X, \Omega_{X}^{1}\right)
$$

defined by $\xi \longmapsto \mathcal{A}^{*} \xi$, for all $\xi \in H^{0}\left(\mathrm{~A}(X), \Omega_{\mathrm{A}(X)}^{1}\right)$, is an isomorphism. Hence the homomorphism

$$
\bar{\alpha}: H^{1}\left(\mathrm{~A}(X), \mathcal{O}_{\mathrm{A}(X)}\right)=\overline{H^{0}\left(\mathrm{~A}(X), \Omega_{\mathrm{A}(X)}^{1}\right)} \longrightarrow \overline{H^{0}\left(X, \Omega_{X}^{1}\right)}=H^{1}\left(X, \mathcal{O}_{X}\right)
$$

is an isomorphism.

In view of the isomorphism $\alpha$ in (3.7) we conclude that there is a natural bijection between the holomorphic connections on $\mathcal{O}_{X}^{\oplus r}$ and the holomorphic connections on $\mathcal{O}_{\mathrm{A}(X)}^{\oplus r}$; 
this isomorphism sends a holomorphic connection $D$ on $\mathcal{O}_{\mathrm{A}(X)}^{\oplus r}$ to the holomorphic connection $\mathcal{A}^{*} D$ on $\mathcal{O}_{X}^{\oplus r}=\mathcal{A}^{*} \mathcal{O}_{\mathrm{A}(X)}^{\oplus r}$.

If $D$ is integrable, then $\mathcal{A}^{*} D$ is integrable. Notice that the converse need not be true in general. To construct examples of non-integrable $D$ such that $\mathcal{A}^{*} D$ is integrable, we recall that there are examples of $(M, \mathbb{D})$, where $M$ is a compact Riemann surface and $\mathbb{D}$ is an irreducible holomorphic connection on $\mathcal{O}_{M}^{\oplus 2}[\mathrm{CDHL}],[\mathrm{BD}],[\mathrm{BDH}]$ (any holomorphic connection on a Riemann surface is automatically integrable). Since the fundamental group of a compact complex torus $\mathbb{T}$ is abelian, there is no irreducible integrable connection on a rank two bundle over $\mathbb{T}$. Also, the pullback of a reducible connection is reducible. Therefore, for any $(M, \mathbb{D})$ as above, the corresponding holomorphic connection on the Albanese variety $\mathrm{A}(M)$ is not integrable.

Note that $\Gamma$ in $(3.5)$ is a quotient of $\pi_{1}\left(X, x_{0}\right)$, because $H_{1}(X, \mathbb{Z})$ is a quotient of $\pi_{1}\left(X, x_{0}\right)$, and $\Gamma$ is a quotient of $H_{1}(X, \mathbb{Z})$.

Proposition 3.10. Let $D$ be an integrable holomorphic connection on $\mathcal{O}_{X}^{\oplus r}$. Then there is an integrable holomorphic connection $\widetilde{D}$ on $\mathcal{O}_{\mathrm{A}(X)}^{\oplus r}$ such that

$$
\left(\mathcal{O}_{X}^{\oplus r}, D\right)=\left(\mathcal{A}^{*} \mathcal{O}_{\mathrm{A}(X)}^{\oplus r}, \mathcal{A}^{*} \widetilde{D}\right)
$$

where $\mathcal{A}$ is the map in (3.6), if and only if the monodromy representation

$$
\rho_{D}: \pi_{1}\left(X, x_{0}\right) \longrightarrow \mathrm{GL}(r, \mathbb{C})
$$

for $D$ factors through the quotient group $\Gamma$ of $\pi_{1}\left(X, x_{0}\right)$.

Proof. We have $\pi_{1}(\mathrm{~A}(X))=\Gamma$, and the homomorphism

$$
\mathcal{A}_{*}: \pi_{1}\left(X, x_{0}\right) \longrightarrow \pi_{1}(\mathrm{~A}(X))
$$

induced by the Albanese map $\mathcal{A}$ in (3.6) actually coincides with the quotient homomorphism $\pi_{1}\left(X, x_{0}\right) \longrightarrow \Gamma$. Therefore, if

$$
\left(\mathcal{O}_{X}^{\oplus r}, D\right)=\left(\mathcal{A}^{*} \mathcal{O}_{\mathrm{A}(X)}^{\oplus r}, \mathcal{A}^{*} \widetilde{D}\right)
$$

for some integrable holomorphic connection $\widetilde{D}$ on $\mathcal{O}_{\mathrm{A}(X)}^{\oplus r}$, then the monodromy representation $\rho_{D}: \pi_{1}\left(X, x_{0}\right) \longrightarrow \mathrm{GL}(r, \mathbb{C})$ for $D$ factors through the quotient group $\Gamma$ of $\pi_{1}\left(X, x_{0}\right)$.

To prove the converse, assume that the monodromy representation $\rho_{D}: \pi_{1}\left(X, x_{0}\right) \longrightarrow$ $\mathrm{GL}(r, \mathbb{C})$ for $D$ factors through the quotient group $\Gamma$ of $\pi_{1}\left(X, x_{0}\right)$. Therefore, the representation

$$
\rho_{D}^{\prime}: \Gamma=\pi_{1}\left(\mathrm{~A}(X), \mathcal{A}\left(x_{0}\right)\right) \longrightarrow \mathrm{GL}(r, \mathbb{C})
$$

given by $\rho_{D}$ produces a pair $(V, \widetilde{D})$, where

- $V$ is a holomorphic vector bundle of rank $r$ on $\mathrm{A}(X)$,

- $\widetilde{D}$ is an integrable holomorphic connection on $V$, and

- $\left(\mathcal{A}^{*} V, \mathcal{A}^{*} \widetilde{D}\right)=\left(\mathcal{O}_{X}^{\oplus r}, D\right)$. 
Therefore, to prove the proposition it suffices to show that

$$
V \simeq \mathcal{O}_{\mathrm{A}(X)}^{\oplus r} .
$$

Consider $\mathbb{C}^{r}$ as a $\Gamma$-module using $\rho_{D}^{\prime}($ in $(3.9))$ and the standard action of $\operatorname{GL}(r, \mathbb{C})$ on $\mathbb{C}^{r}$ Since $\Gamma$ is an abelian group, we contend that the $\Gamma$-module $\mathbb{C}^{r}$ decomposes as

$$
\mathbb{C}^{r}=\bigoplus_{i=1}^{m} L_{i} \otimes U_{i},
$$

where $L_{i}$ (respectively, $U_{i}$ ) is a one-dimensional (respectively, unipotent) representation of $\Gamma$. Indeed, let $S$ be an indecomposable summand of the $\Gamma$-module $\mathbb{C}^{r}$ and, for any given $\gamma \in \Gamma$, consider the decomposition of $S$ into generalized eigenspaces: $\bigoplus_{i} S_{i}$. Since every $\delta \in \Gamma$ commutes with $\gamma$, we can say that every $S_{i}$ is invariant under $\Gamma$; it follows that $\gamma$ has a single eigenvalue in $S$ (recall that $S$ is indecomposable). Associating to each $\gamma$ the previous eigenvalue, we get a homomorphism $\Gamma \longrightarrow \mathbb{C}^{*}$ and hence a one dimensional representation $L$. Now, $L^{\vee} \otimes S$ is indecomposable and has only one eigenvalue, namely 1 ; here $L^{\vee}$ denotes the dual of $L$. Simultaneous triangularization — which follows easily from the fact that $\Gamma$ is abelian - now shows that $L^{\vee} \otimes S$ is unipotent thus establishing the decomposition in (3.11).

From (3.11) we obtain a decomposition of the connection $(V, \widetilde{D})$ as

$$
V=\bigoplus_{i=1}^{m} \mathcal{L}_{i} \otimes \mathcal{U}_{i},
$$

where $\mathcal{L}_{i}$ stands for a rank one integrable connection and $\mathcal{U}_{i}$ for a unipotent one. Note that, since $\mathcal{U}_{i}$ is unipotent, each $\mathcal{L}_{i}$ is a sub-connection of $V$ and hence $\mathcal{A}^{*} \mathcal{L}_{i}$ is a subconnection of $\mathcal{A}^{*} V$. Using a dualization and Proposition 3.2, we can say that the vector bundle underlying $\mathcal{A}^{*} \mathcal{L}_{i}$ is trivial. Since the homomorphism

$$
\operatorname{Pic}^{0}(\mathrm{~A}(X)) \longrightarrow \operatorname{Pic}^{0}(X), L \longmapsto \mathcal{A}^{*} L
$$

where $\mathcal{A}$ is the map in (3.6), is an isomorphism, we conclude that

the underlying holomorphic line bundle of $\mathcal{L}_{i}$ in (3.12) is trivial

for all $1 \leq i \leq m$.

Next, we note that the connection $\mathcal{A}^{*} \mathcal{U}_{i}$ is a sub-connection of $\mathcal{A}^{*}(V) \otimes\left(\mathcal{A}^{*} \mathcal{L}_{i}^{\vee}\right)$ and hence, applying a dualization and Proposition 3.2, from (3.13) we conclude that $\mathcal{A}^{*} \mathcal{U}_{i} \in$ $\mathcal{T}_{d R}(X)$. Now, using that the homomorphism

$$
H^{1}\left(\mathrm{~A}(X), \mathcal{O}_{\mathrm{A}(X)}\right) \longrightarrow H^{1}\left(X, \mathcal{O}_{X}\right), \quad \theta \longmapsto \mathcal{A}^{*} \theta
$$

is an isomorphism (see (3.8)), and that extensions of $\mathcal{O}_{\mathrm{A}(X)}$ by $\mathcal{O}_{\mathrm{A}(X)}$ are parametrized by the cohomology space $H^{1}\left(\mathrm{~A}(X), \mathcal{O}_{\mathrm{A}(X)}\right)$, a straightforward induction argument proves that $\mathcal{U}_{i}$ is trivial. This shows that $\mathcal{U}_{i} \in \mathcal{T}_{d R}(\mathrm{~A}(X))$.

Let us now study the case of "abelian" Kähler manifolds by first recalling certain fundamental facts from the theory of affine group schemes. 
Let $U$ be an algebraic affine and unipotent group scheme over $\mathbb{C}$ [Wa, 8.3]: there is a closed immersion of $U$ into some group of strict upper triangular matrices. Endowing the nilpotent Lie algebra $\operatorname{Lie}(U)$ with its Baker-Campbell-Hausdorff multiplication, it is known that

$$
\exp : \operatorname{Lie}(U) \longrightarrow U
$$

is an isomorphism of group schemes [Ho, Theorem XVII.4.2, p. 232.]; in particular, if $U$ is in addition a commutative group scheme, then $U \simeq \mathbb{G}_{a}^{r}$ for some $r$. Moreover, $r=\operatorname{dim}_{\mathbb{C}} \operatorname{Hom}\left(U, \mathbb{G}_{a}\right)$ [Wa, Theorem 8.4]. Said differently,

$$
U \simeq \operatorname{Hom}\left(U, \mathbb{G}_{a}\right) .
$$

Still in the topic of affine group schemes, for any given abstract abelian group $\Lambda$, we shall denote by $\operatorname{Diag}(\Lambda)$ the diagonalizable group scheme corresponding to the abstract group $\Lambda$ as explained in [Wa, 2.2]; on the level of $\mathbb{C}$-points, $\operatorname{Diag}(\Lambda)$ is just $\operatorname{Hom}\left(\Lambda, \mathbb{C}^{\times}\right)$.

Proposition 3.11. Suppose that $\pi_{1}\left(X, x_{0}\right)$ is abelian. Then

$$
\Theta\left(X, x_{0}\right) \simeq H^{0}\left(X, \Omega_{X}^{1}\right) \times \operatorname{Diag}\left(H^{0}\left(X, \Omega_{X}^{1}\right)\right)
$$

Proof. Since $\pi_{1}\left(X, x_{0}\right)$ is abelian, the group scheme $\varpi\left(X, x_{0}\right)$ is abelian. This implies that $\Theta\left(X, x_{0}\right)$ is abelian and hence is a product of a unipotent $U$ and a diagonal $D$ group scheme [Wa, p. 70, Theorem, 9.5]. The arguments made in Lemma 3.5 and Lemma 3.6 jointly with the preliminary material on group schemes recalled above now allow us to explicitly determine $U$ and $D$ as wanted. Indeed, the group of characters of $U \times D$ (respectively, additive characters) is simply the group of characters of $D$ (respectively, additive characters of $U$ ) as explained in [Wa, Chapter 8], Corollary in 8.3 and Exercise 6.

\section{RiEMANN SURFACES AND COMPACT COMPLEX TORI}

4.1. Neutral Tannakian category for a Riemann surface. Let $X$ and $Y$ be two compact connected Riemann surfaces of common genus $g$, with $g \geq 1$. Fix a point $x_{0} \in X$. Let

$$
\beta: X \longrightarrow Y
$$

be a $C^{\infty}$ orientation preserving diffeomorphism. Let

$$
\widehat{\beta}: \pi_{1}\left(X, x_{0}\right) \longrightarrow \pi_{1}\left(Y, \beta\left(x_{0}\right)\right)
$$

be the homomorphism of fundamental groups induced by $\beta$. It produces a unique algebraic homomorphism

$$
\beta_{\natural}: \varpi\left(X, x_{0}\right) \longrightarrow \varpi\left(Y, \beta\left(x_{0}\right)\right)
$$

such that $\beta_{\natural} \circ \phi=\phi_{Y} \circ \widehat{\beta}$, where $\phi$ is the homomorphism in (2.1) and

$$
\phi_{Y}: \pi_{1}\left(Y, \beta\left(x_{0}\right)\right) \longrightarrow \varpi\left(Y, \beta\left(x_{0}\right)\right)
$$

is the similar homomorphism for $Y$. 
We will say that the homomorphism $\beta_{\natural}$ in (4.2) descends to a homomorphism from $\Theta\left(X, x_{0}\right)$, constructed in $(3.1)$, to $\Theta\left(Y, \beta\left(x_{0}\right)\right)$ if there is a homomorphism

$$
\beta_{\natural}^{\prime}: \Theta\left(X, x_{0}\right) \longrightarrow \Theta\left(Y, \beta\left(x_{0}\right)\right)
$$

such that

$$
\beta_{\natural}^{\prime} \circ \mathbf{q}_{X}=\mathbf{q}_{Y} \circ \beta_{\natural},
$$

where $\mathbf{q}_{X}$ is the homomorphism in (3.2) and $\mathbf{q}_{Y}: \varpi\left(Y, \beta\left(x_{0}\right)\right) \longrightarrow \Theta\left(Y, \beta\left(x_{0}\right)\right)$ is the similar homomorphism for $Y$, while $\beta_{\natural}$ is constructed in (4.2).

Theorem 4.1. Assume that the homomorphism $\beta_{\natural}$ in (4.2) descends to a homomorphism from $\Theta\left(X, x_{0}\right)$ to $\Theta\left(Y, \beta\left(x_{0}\right)\right)$. Then the two Riemann surfaces $X$ and $Y$ are isomorphic.

Proof. Let $\beta_{\natural}^{\prime}: \Theta\left(X, x_{0}\right) \longrightarrow \Theta\left(Y, \beta\left(x_{0}\right)\right)$ be a homomorphism such that the diagram

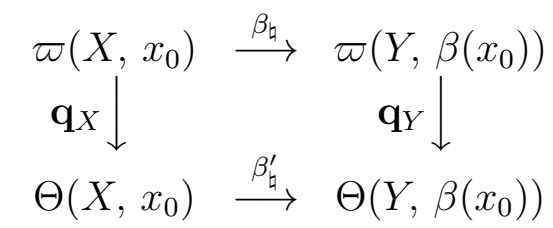

is commutative. Let

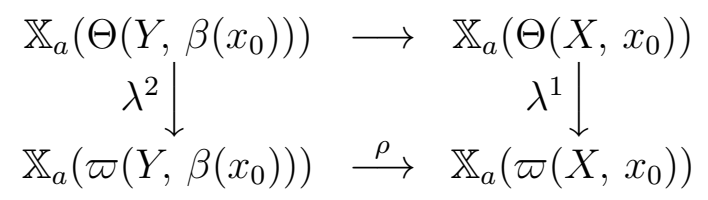

be the corresponding commutative diagram of vector spaces (see Section 3.2).

Now, according to Lemma 3.6,

$$
\mathbb{X}_{a}\left(\Theta\left(X, x_{0}\right)\right)=H^{0}\left(X, \Omega_{X}^{1}\right) \quad \text { and } \quad \mathbb{X}_{a}\left(\varpi\left(X, x_{0}\right)\right)=H_{d R}^{1}(X, \mathbb{C}) .
$$

Similarly, we have

$$
\mathbb{X}_{a}\left(\Theta\left(Y, \beta\left(x_{0}\right)\right)\right)=H^{0}\left(Y, \Omega_{Y}^{1}\right) \quad \text { and } \quad \mathbb{X}_{a}\left(\varpi\left(Y, \beta\left(x_{0}\right)\right)\right)=H_{d R}^{1}(Y, \mathbb{C}) .
$$

The homomorphism $\lambda^{1}$ in (4.4) is the natural inclusion of $H^{0}\left(X, \Omega_{X}^{1}\right)$ in $H_{d R}^{1}(X, \mathbb{C})$ given by the fact that any holomorphic 1 -form on $X$ is closed. The homomorphism

$$
\rho: H_{d R}^{1}(Y, \mathbb{C})=\mathbb{X}_{a}\left(\varpi\left(Y, \beta\left(x_{0}\right)\right)\right) \longrightarrow \mathbb{X}_{a}\left(\varpi\left(X, x_{0}\right)\right)=H_{d R}^{1}(X, \mathbb{C})
$$

in (4.4) coincides with the pullback homomorphism

$$
\beta^{*}: H_{d R}^{1}(Y, \mathbb{C}) \longrightarrow H_{d R}^{1}(X, \mathbb{C}), c \longmapsto \beta^{*} c,
$$

where $\beta$ is diffeomorphism in (4.1).

For the isomorphism $\beta^{*}$ in (4.6), we have

$$
\beta^{*}\left(H^{1}(Y, \mathbb{Z})\right)=H^{1}(X, \mathbb{Z}) .
$$

Furthermore, since the diffeomorphism $\beta$ is orientation preserving, it takes the natural symplectic pairing on $H_{d R}^{1}(Y, \mathbb{C})$ defined by

$$
c_{1} \otimes c_{2} \longmapsto \int_{Y} c_{1} \wedge c_{2} \in \mathbb{C}
$$


to the corresponding symplectic pairing on $H_{d R}^{1}(X, \mathbb{C})$.

The Jacobian $J(Y)$ of $Y$ coincides with the following quotient:

$$
\left(H_{d R}^{1}(Y, \mathbb{C}) / H^{0}\left(Y, \Omega_{Y}^{1}\right)\right) / H^{1}(Y, \mathbb{Z})=J(Y),
$$

and the natural principal polarization on $J(Y)$ is constructed using the pairing in (4.7) and the complex structure of $H_{d R}^{1}(Y, \mathbb{C})$. More precisely, the holomorphic tangent bundle $T J(Y)$ of $J(Y)$ is the trivial holomorphic bundle $J(Y) \times \overline{H^{0}\left(Y, \Omega_{Y}^{1}\right)} \longrightarrow J(Y)$ with fiber $\overline{H^{0}\left(Y, \Omega_{Y}^{1}\right)}=H^{1}\left(Y, \mathcal{O}_{Y}\right)$. The Hermitian form on $\overline{H^{0}\left(Y, \Omega_{Y}^{1}\right)}$ defined by

$$
\bar{c}_{1} \otimes \bar{c}_{2} \longmapsto-\sqrt{-1} \int_{Y} \bar{c}_{1} \wedge c_{2} \in \mathbb{C}, c_{1}, c_{2} \in H^{0}\left(Y, \Omega_{Y}^{1}\right)
$$

produces the canonical principal polarization on $J(Y)$.

We noted above that the $\mathbb{C}$-linear isomorphism $\beta^{*}$ in $(4.6)$ takes $H^{1}(Y, \mathbb{Z})$ to isomorphically $H^{1}(X, \mathbb{Z})$ and takes the symplectic pairing in $(4.7)$ to the corresponding pairing for $X$. Since

$$
\rho=\beta^{*},
$$

where $\rho$ is the homomorphism in (4.5) (and (4.4)), from the commutativity of the diagram in (4.4) we now conclude that $J(Y)$ is isomorphic to the Jacobian $J(X)$ of $X$ as a principally polarized abelian variety. Now from the standard Torelli theorem (see [ACGH, Ch. VI, $\S 3$, pp. 245-246]) we conclude that $X$ is isomorphic to $Y$.

4.2. Neutral Tannakian category for a compact complex torus. Let $\mathbb{T}$ be a compact complex torus of complex dimension $d$. The group scheme $\varpi\left(\mathbb{T}, x_{0}\right)$ is abelian because $\pi_{1}\left(\mathbb{T}, x_{0}\right)$ is so. Hence the quotient $\Theta\left(X, x_{0}\right)$ of $\varpi\left(\mathbb{T}, x_{0}\right)$ is also abelian. As in the proof of Theorem 4.1 we consider the corresponding additive character spaces $\mathbb{X}_{a}\left(\varpi\left(\mathbb{T}, x_{0}\right)\right)$ and $\mathbb{X}_{a}\left(\Theta\left(\mathbb{T}, x_{0}\right)\right)$ and their Lie algebras. From Lemma 3.6 we have

$$
\mathbb{X}_{a}\left(\varpi\left(\mathbb{T}, x_{0}\right)\right)=H_{d R}^{1}(\mathbb{T}, \mathbb{C})
$$

and

$$
\mathbb{X}_{a}\left(\Theta\left(\mathbb{T}, x_{0}\right)\right)=H^{0}\left(\mathbb{T}, \Omega_{\mathbb{T}}^{1}\right),
$$

where $\Omega_{\mathbb{T}}^{1}$ is the holomorphic cotangent bundle of $\mathbb{T}$. Consider the linear map

$$
\Psi: H^{0}\left(\mathbb{T}, \Omega_{\mathbb{T}}^{1}\right)=\mathbb{X}_{a}\left(\Theta\left(\mathbb{T}, x_{0}\right)\right) \longrightarrow \mathbb{X}_{a}\left(\varpi\left(\mathbb{T}, x_{0}\right)\right)=H_{d R}^{1}(\mathbb{T}, \mathbb{C})
$$

induced by the homomorphism $\mathbf{q}_{\mathbb{T}}$ in (3.2). We note that $\Psi$ coincides with the natural inclusion of $H^{0}\left(\mathbb{T}, \Omega_{\mathbb{T}}^{1}\right)$ in $H_{d R}^{1}(\mathbb{T}, \mathbb{C})$ given by the fact that any holomorphic 1 -form on $\mathbb{T}$ is closed.

Let $\mathbb{S}$ be a compact complex torus of complex dimension $d$, and let

$$
\varphi: \mathbb{T} \longrightarrow \mathbb{S}
$$

be a diffeomorphism. Let

$$
\varphi_{\natural}: \varpi\left(\mathbb{T}, x_{0}\right) \longrightarrow \varpi\left(\mathbb{S}, \varphi\left(x_{0}\right)\right)
$$


be the homomorphism corresponding to $\varphi$. We say that $\varphi_{\natural}$ descends to a homomorphism from $\Theta\left(\mathbb{T}, x_{0}\right)$ to $\Theta\left(\mathbb{S}, \varphi\left(x_{0}\right)\right)$ if there is a homomorphism

$$
\varphi_{\natural}^{\prime}: \Theta\left(\mathbb{T}, x_{0}\right) \longrightarrow \Theta\left(\mathbb{S}, \varphi\left(x_{0}\right)\right)
$$

such that the diagram

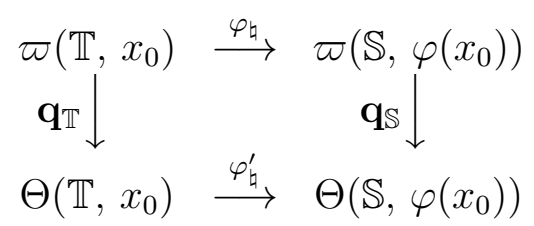

is commutative, where $\mathbf{q}_{\mathbb{T}}$ and $\mathbf{q}_{\mathbb{S}}$ are the projections in (3.2).

Proposition 4.2. Let $\varphi: \mathbb{T} \longrightarrow \mathbb{S}$ be a diffeomorphism such that the homomorphism $\varphi_{\sharp}$ in (4.9) descends to a homomorphism from $\Theta\left(\mathbb{T}, x_{0}\right)$ to $\Theta\left(\mathbb{S}, \varphi\left(x_{0}\right)\right)$. Then there is a biholomorphism

$$
\widetilde{\varphi}: \mathbb{T} \longrightarrow \mathbb{S}
$$

which is homotopic to the map $\varphi$.

Proof. Let $\varphi_{\natural}^{\prime}: \Theta\left(\mathbb{T}, x_{0}\right) \longrightarrow \Theta\left(\mathbb{S}, \varphi\left(x_{0}\right)\right)$ be the homomorphism such the diagram in (4.10) is commutative. Let

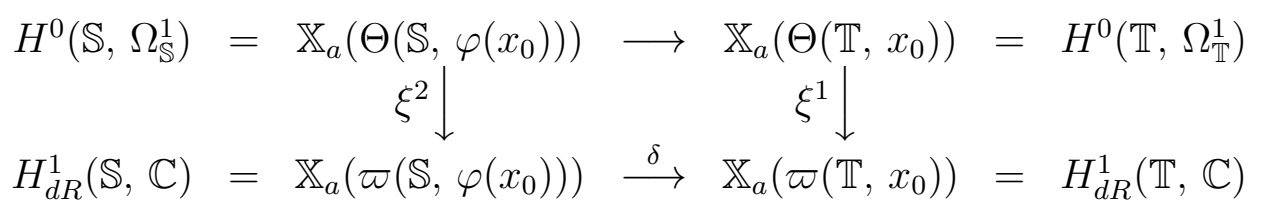

be the commutative diagram of $\mathbb{C}$-linear maps corresponding to (4.10).

Let

$$
\varphi^{*}: H^{1}(\mathbb{S}, \mathbb{C}) \longrightarrow H^{1}(\mathbb{T}, \mathbb{C}), \quad c \longmapsto \varphi^{*} c
$$

be the pullback map. The homomorphism $\delta$ in (4.11) coincides with the homomorphism $\varphi^{*}$ in (4.12). Therefore, from (4.11) we conclude that

$$
\varphi^{*}\left(H^{0}\left(\mathbb{S}, \Omega_{\mathbb{S}}^{1}\right)\right)=H^{0}\left(\mathbb{T}, \Omega_{\mathbb{T}}^{1}\right) .
$$

We also have

$$
\varphi^{*}\left(H^{1}(\mathbb{S}, \mathbb{Z})\right)=H^{1}(\mathbb{T}, \mathbb{Z})
$$

because $\varphi$ is a diffeomorphism.

We consider $\mathbb{T}$ (respectively, $\mathbb{S}$ ) as a complex abelian Lie group by taking $x_{0}$ (respectively, $\varphi\left(x_{0}\right)$ ) to be the identity element of $\mathbb{T}$ (respectively, $\mathbb{S}$ ). From (4.13) and (4.14) it follows immediately that the homomorphism $\varphi^{*}$ in (4.12) induces a holomorphic isomorphism

$$
\begin{aligned}
& \widetilde{\varphi}^{\vee}: \mathbb{S}^{\vee}:=\left(H_{d R}^{1}(\mathbb{S}, \mathbb{C}) / H^{0}\left(\mathbb{S}, \Omega_{\mathbb{S}}^{1}\right)\right) / H^{1}(\mathbb{S}, \mathbb{Z}) \\
& \longrightarrow\left(H_{d R}^{1}(\mathbb{T}, \mathbb{C}) / H^{0}\left(\mathbb{T}, \Omega_{\mathbb{T}}^{1}\right)\right) / H^{1}(\mathbb{T}, \mathbb{Z})=\mathbb{T}^{\vee}
\end{aligned}
$$

here $\mathbb{S}^{\vee}=\operatorname{Pic}^{0}(\mathbb{S})$ is the dual torus of $\mathbb{S}$, and $\mathbb{T}^{\vee}=\operatorname{Pic}^{0}(\mathbb{T})$ is the dual torus of $\mathbb{T}$. Let

$$
\widetilde{\varphi}: \mathbb{T}=\left(\mathbb{T}^{\vee}\right)^{\vee} \longrightarrow\left(\mathbb{S}^{\vee}\right)^{\vee}=\mathbb{S}
$$

be the dual of the above homomorphism $\widetilde{\varphi}^{\vee}$. 
From the construction of the above homomorphism $\widetilde{\varphi}$ it is evident that the pullback homomorphism

$$
\widetilde{\varphi}^{*}: H_{d R}^{1}(\mathbb{S}, \mathbb{C}) \longrightarrow H_{d R}^{1}(\mathbb{T}, \mathbb{C}), \quad c \longmapsto \widetilde{\varphi}^{*} c
$$

coincides with the homomorphism $\varphi^{*}$ in (4.12). This implies that the two maps $\widetilde{\varphi}$ and $\varphi$ are homotopic.

\section{Completely decomposable vector Bundles}

As before, $X$ is a compact connected Kähler manifold. We define and study in this section a subcategory of $\mathcal{C}_{d R}(X)$ given by the following:

Definition 5.1. Let $\mathcal{D}_{d R}(X)$ be the full subcategory of $\mathcal{C}_{d R}(X)$ whose objects are pairs $(E, D)$ satisfying the condition that the holomorphic vector bundle $E$ is a direct sum of holomorphic line bundles.

It is straightforward to check that $\mathcal{D}_{d R}(X)$ is stable under tensor products and duals. In order to prove that $\mathcal{D}_{d R}(X)$ is abelian we will need the next two lemmas.

Lemma 5.2. Let $L$ be a holomorphic line bundle on $X$. Then $L$ admits a holomorphic connection if and only if $c_{1}(L)=0$. If $L$ admits a holomorphic connection, then any holomorphic connection on $L$ is integrable.

Let $L_{i}, 1 \leq i \leq r$, be holomorphic line bundles on $X$ such that the holomorphic vector bundle $\bigoplus_{i=1}^{r} L_{i}$ admits a holomorphic connection. Then each $L_{i}$ admits a holomorphic connection; in other words, $c_{1}\left(L_{i}\right)=0$.

Proof. Since $X$ is Kähler, if $L$ admits a holomorphic connection, then $c_{1}(L)=0$ [At, pp. 192-193, Theorem 4]. If $c_{1}(L)=0$, then $L$ admits an integrable holomorphic connection $\nabla^{L}$ whose monodromy lies in U(1) see [LT, Corollary 1.3.12, p.40]; note also that any holomorphic line bundle admits a Hermitian-Einstein metric [UY], [LT, p. 61, Theorem 3.0.1].

If $L$ admits a (integrable) holomorphic connection $\nabla^{L}$, then any holomorphic connection on $L$ is of the form $\nabla^{L}+\beta$, where $\beta \in H^{0}\left(X, \Omega_{X}^{1}\right)$. The curvature of $\nabla^{L}+\beta$ is $\mathrm{d} \beta$, because $\nabla^{L}$ is integrable. Since any holomorphic 1 -form on $X$ is closed, we conclude that $\nabla^{L}+\beta$ is integrable.

Let $p_{j}: \bigoplus_{i=1}^{r} L_{i} \longrightarrow L_{j}$ be the projection to the $j$-th factor. Then for any holomorphic connection $D$ on $\bigoplus_{i=1}^{r} L_{i}$, the composition of homomorphisms

$$
L_{j} \hookrightarrow \bigoplus_{i=1}^{r} L_{i} \stackrel{D}{\longrightarrow}\left(\bigoplus_{i=1}^{r} L_{i}\right) \otimes \Omega_{X}^{1} \stackrel{p_{j} \otimes \mathrm{Id}_{\Omega_{X}^{1}}}{\longrightarrow} L_{j} \otimes \Omega_{X}^{1}
$$

is a holomorphic connection on $L_{j}$. 
Lemma 5.3. Let $L_{i}, 1 \leq i \leq r$, and $\mathcal{L}_{j}, 1 \leq j \leq \ell$, be holomorphic line bundles on $X$ such that $c_{i}\left(L_{i}\right)=0=c_{1}\left(\mathcal{L}_{j}\right)$ for all $i, j$. Let

$$
f: \bigoplus_{i=1}^{r} L_{i} \longrightarrow \bigoplus_{j=1}^{\ell} \mathcal{L}_{j}
$$

be a holomorphic homomorphism. Then the following two hold:

(1) If $\operatorname{kernel}(f) \neq 0$, then $\operatorname{kernel}(f)$ is a direct sum of holomorphic line bundles of vanishing first Chern class.

(2) If cokernel $(f) \neq 0$, then cokernel $(f)$ is a direct sum of holomorphic line bundles of vanishing first Chern class.

Proof. Equip each $L_{i}$ (respectively, $\mathcal{L}_{j}$ ) with an integrable holomorphic connection $\nabla^{i}$ (respectively, $\widetilde{\nabla}^{j}$ ) such that the monodromy lies in U(1). The holomorphic homomorphism $f$ intertwines the connections $\bigoplus_{i=1}^{r} \nabla^{i}$ on $\bigoplus_{i=1}^{r} L_{i}$ and $\bigoplus_{j=1}^{\ell} \widetilde{\nabla}^{j}$ on $\bigoplus_{j=1}^{\ell} \mathcal{L}_{j}$ [LT, p. 50, Theorem 2.2.1]. Consequently, $\operatorname{kernel}(f)$ is a holomorphic subbundle of $\bigoplus_{i=1}^{r} L_{i}$ preserved by $\bigoplus_{i=1}^{r} \nabla^{i}$, and cokernel $(f)$ is a quotient bundle of $\bigoplus_{j=1}^{\ell} \mathcal{L}_{j}$ on which $\bigoplus_{j=1}^{\ell} \widetilde{\nabla}^{j}$ induces a holomorphic connection. This implies that

$$
\text { degree }(\operatorname{kernel}(f))=0=\operatorname{degree}(\operatorname{cokernel}(f))
$$

(see Lemma 5.2).

Since $\bigoplus_{i=1}^{r} L_{i}$ is polystable of degree zero, and it is a direct sum of line bundles, from the observation in (5.1) that degree $(\operatorname{kernel}(f))=0$ it follows that $\operatorname{kernel}(f)$ is a direct sum of holomorphic line bundles of degree zero. Since $\bigoplus_{j=1}^{\ell} \mathcal{L}_{j}$ is polystable of degree zero, and it is a direct sum of line bundles (if it is nonzero), from the observation in (5.1) that degree $(\operatorname{cokernel}(f))=0$ it follows that cokernel $(f)$ is a direct sum of holomorphic line bundles of degree zero (if it is nonzero).

Using Lemma 5.3 it can be deduced that the category $\mathcal{D}_{d R}(X)$ is abelian.

Fix a point $x_{0} \in X$. Equip the category $\mathcal{D}_{d R}(X)$ with the exact functor to the category of finite dimensional complex vector spaces defined by $\left.(E, D) \longmapsto E\right|_{x_{0}}$. Let

$$
\Delta\left(X, x_{0}\right)
$$

be the affine group scheme over $\mathbb{C}$ corresponding to $\mathcal{D}_{d R}(X)$ [DMOS, p. 130, Theorem 2.11]. Note that $\Delta\left(X, x_{0}\right)$ is the target of a morphism of affine group schemes

$$
\varpi\left(X, x_{0}\right) \longrightarrow \Delta\left(X, x_{0}\right)
$$

which, according to the the standard criterion [DMOS, Proposition 2.21, p.139] amplified by [BHdS, Lemma 2.1], and Lemma 5.3 is a quotient morphism.

The homomorphism $\mathbf{q}_{X}$ in (3.2) factors through the quotient $\Delta\left(X, x_{0}\right)$ and gives a quotient homomorphism

$$
\mathbf{p}_{X}: \Delta\left(X, x_{0}\right) \longrightarrow \Theta\left(X, x_{0}\right)
$$


For a pointed compact Kähler manifold $\left(Y, y_{0}\right)$ it can be shown that the natural homomorphism

$$
\Delta\left(X, x_{0}\right) \times \Delta\left(Y, y_{0}\right) \longrightarrow \Delta\left(X \times Y,\left(x_{0}, y_{0}\right)\right)
$$

is an isomorphism; it's proof is similar to that of Proposition 3.9.

The homomorphism of additive character $\mathbb{C}$-vector spaces

$$
\mathbb{X}_{a}\left(\Theta\left(X, x_{0}\right)\right) \longrightarrow \mathbb{X}_{a}\left(\Delta\left(X, x_{0}\right)\right)
$$

induced by $\mathbf{p}_{X}$ in (5.4) is an isomorphism as follows from Lemma 5.3 and the interpretation of these vector spaces as extension groups (see Section 3.2). In view of this, examining the proofs of Theorem 4.1 and Proposition 4.2 we conclude that these two results remain valid if $\Theta\left(X, x_{0}\right)$ is replaced by the group scheme $\Delta\left(X, x_{0}\right)$ in (5.2).

\section{Connections on PSeudostable Vector Bundles}

We shall now relate the group scheme $\Theta\left(X, x_{0}\right)$ of Section 3.1 to another group scheme which deals only with a full subcategory of vector bundles.

Definition 6.1. A holomorphic vector bundle $E$ over $X$ is said to be pseudostable (see $[\mathrm{BG}])$ if the object Higgs vector bundle $(E, 0)$ lies in the category $\mathcal{C}_{D o l}(X)$ introduced in Section 2. To wit,

- degree $(E)=0$,

- $c h_{2}(E) \wedge \omega^{d-2}=0$, and

- there exists a filtration of $E$ by holomorphic subbundles

$$
0=E_{0} \subset E_{1} \subset \cdots \subset E_{\ell-1} \subset E_{\ell}=E
$$

in which every $E_{j} / E_{j-1}, 1 \leq j \leq \ell$, is stable and of degree zero.

(Actually the third condition implies the first condition.) The full subcategory of pseudostable vector bundles on $X$ will be denoted by $\operatorname{Vect}_{0}^{\mathrm{s}}(X)$.

The following theorem, which is based on the works of Simpson and Corlette, is proved in [Si2] (see [Si2, pp. 35-37, (3.4.1)-(3.4.5)] and [Si2, p. 70]).

Theorem 6.2 ([Si2, (3.4.1)-(3.4.5)]).

(1) The category $\operatorname{Vect}_{0}^{\mathrm{s}}(X)$ is abelian and stable under the tensor product of vector bundles.

(2) Any $E \in \operatorname{Vect}_{0}^{\mathrm{s}}(X)$ carries a canonical holomorphic integrable connection.

(3) If $X$ is projective, and the cohomology class of $\omega$ is rational, then $\operatorname{Vect}_{0}^{\mathrm{s}}(X)$ coincides with the category of all semistable vector bundles $E$ such that $h_{1}(E) \wedge \omega^{d-1}=$ $c h_{2}(E) \wedge \omega^{d-2}=0$ (see [Si2, p. 39, Theorem 2] for it).

Remark 6.3. As mentioned in Section 2, the category $\mathcal{C}_{D o l}(X)$ is equivalent to $\mathcal{C}_{d R}(X)$ [Si2, p. 36, Lemma 3.5]. Take $(E, \theta) \in \mathcal{C}_{D o l}(X)$, and let $(V, \nabla) \in \mathcal{C}_{d R}(X)$ be the object corresponding to $(E, \theta)$ by the equivalence of categories between $\mathcal{C}_{D o l}(X)$ and $\mathcal{C}_{d R}(X)$. Although the $C^{\infty}$ vector bundles underlying $E$ and $V$ coincide, their holomorphic structure 
do not coincide in general. However, when $\theta=0$, then the holomorphic structures of $E$ and $V$ coincide. Therefore, $\nabla$ is a holomorphic integrable connection on $V=E$. We note that when $E$ is polystable, then this holomorphic connection $\nabla$ on $E$ coincides with the unique holomorphic integrable connection on $E$ whose monodromy is unitary. See [BS, p. 4004, Theorem 3.1] for a generalization of this canonical connection on $E \in \operatorname{Vect}_{0}^{\mathrm{s}}(X)$ to principal bundles.

Remark 6.4. Take $E_{1}, E_{2} \in \operatorname{Vect}_{0}^{\mathrm{s}}(X)$. The canonical holomorphic integrable connections on $E_{1}$ and $E_{2}$ (see Remark 6.3 ) will be denoted by $\nabla^{1}$ and $\nabla^{2}$ respectively. Let

$$
\Phi: E_{1} \longrightarrow E_{2}
$$

be a homomorphism of coherent analytic sheaves. Then $\Phi$ is a homomorphism from $\left(E_{1}, 0\right) \in \mathcal{C}_{D o l}(X)$ to $\left(E_{2}, 0\right) \in \mathcal{C}_{D o l}(X)$. Therefore, from the equivalence of categories between $\mathcal{C}_{D o l}(X)$ and $\mathcal{C}_{d R}(X)$ ([Si2, p. 36, Lemma 3.5]) we conclude that $\Phi$ is a homomorphism from $\left(E_{1}, \nabla^{1}\right) \in \mathcal{C}_{d R}(X)$ to $\left(E_{2}, \nabla^{2}\right) \in \mathcal{C}_{d R}(X)$. In particular, $\Phi\left(E_{1}\right) \subset E_{2}$ is preserved by the connection $\nabla^{2}$ on $E_{2}$. Now setting $E_{1}$ to be the trivial line bundle $\mathcal{O}_{X}$ we conclude that every holomorphic section $s \in H^{0}\left(X, E_{2}\right)$ is flat (same as integrable) with respect to the connection $\nabla^{2}$ on $E_{2}$. It also follows that the canonical connection on $E_{1} \oplus E_{2}$ coincides with $\nabla^{1} \oplus \nabla^{2}$. Moreover, the canonical connection on $E_{1} \otimes E_{2}$ (respectively, $\left.\operatorname{Hom}\left(E_{1}, E_{2}\right)\right)$ coincides with the connection on $E_{1} \otimes E_{2}\left(\right.$ respectively, $\operatorname{Hom}\left(E_{1}, E_{2}\right)$ ) induced by $\nabla^{1}$ and $\nabla^{2}$. These properties of the canonical connection were crucial in the proofs of [BS, p. 4004, Theorem 3.1] and [BG, p. 20, Theorem 1.1].

It then follows that there exists an affine group scheme $\pi^{\mathrm{S}}\left(X, x_{0}\right)$ over $\mathbb{C}$ such that the functor

$$
\text { - }\left.\right|_{x_{0}}: \operatorname{Vect}_{0}^{\mathrm{s}}(X) \longrightarrow \operatorname{Vect}_{\mathbb{C}}
$$

induces an equivalence between $\operatorname{Vect}_{0}^{\mathrm{s}}(X)$ and the category of finite dimensional algebraic representations of $\pi^{\mathrm{S}}\left(X, x_{0}\right)$.

Following the path of previous sections, we define $\mathcal{S}_{d R}(X)$ as the full subcategory of $\mathcal{C}_{d R}(X)$ consisting of those $(E, \nabla)$ such that $E$ belongs to $\operatorname{Vect}_{0}^{\mathrm{s}}(X)$. This produces another group scheme

$$
\Sigma\left(X, x_{0}\right)
$$

whose category of representations is, via the functor $\left.\bullet\right|_{x_{0}}$, simply $\mathcal{S}_{d R}(X)$.

Using the forgetful functor

$$
U: \mathcal{S}_{d R}(X) \longrightarrow \operatorname{Vect}_{0}^{\mathrm{s}}(X)
$$

and a the natural inclusion

$$
J: \mathcal{T}_{d R}(X) \longrightarrow \mathcal{S}_{d R}(X)
$$

we arrive at morphisms

$$
\pi^{\mathrm{S}}\left(X, x_{0}\right) \stackrel{\mathbf{u}_{X}}{\longrightarrow} \Sigma\left(X, x_{0}\right) \stackrel{\mathbf{j}_{X}}{\longrightarrow} \Theta\left(X, x_{0}\right)
$$

Clearly, $\mathbf{j}_{X} \circ \mathbf{u}_{X}$ is the trivial homomorphism. 
Theorem 6.5. In (6.2), the morphism $\mathbf{u}_{X}$ is a closed immersion, $\mathbf{j}_{X}$ is a quotient map and the image of $\mathbf{u}_{X}$ is the kernel of $\mathbf{j}_{X}$. In addition, $\mathbf{u}_{X}$ possesses a left inverse

$$
\mathbf{v}_{X}: \Sigma\left(X, x_{0}\right) \longrightarrow \pi^{\mathrm{S}}\left(X, x_{0}\right)
$$

so that

$$
\left(\mathbf{v}_{X}, \mathbf{j}_{X}\right): \Sigma\left(X, x_{0}\right) \longrightarrow \pi^{\mathrm{S}}\left(X, x_{0}\right) \times \Theta\left(X, x_{0}\right)
$$

is an isomorphism.

Proof. The morphism $\mathbf{q}_{X}: \varpi\left(X, x_{0}\right) \longrightarrow \Theta\left(X, x_{0}\right)$ in (3.2) factors through $\mathbf{j}_{X}$ so that the fact that $\mathbf{q}_{X}$ is a quotient morphism shows that $\mathbf{j}_{X}$ is likewise. That $\mathbf{j}_{X}$ is a closed immersion follows from the standard criterion [DMOS, p. 139, Proposition 2.21] and Theorem 6.2.

Let us now prove that $\mathbf{u}_{X}$ identifies $\Sigma\left(X, x_{0}\right)$ with $\operatorname{Ker}\left(\mathbf{q}_{X}\right)$ by verifying conditions (iii)-a, (iii)-b and (iii)-c of Theorem A.1 in [EHS]. Condition (iii)-a is a tautology, while Theorem 6.2 immediately ensures (iii)-c. We then need to assure the validity of (iii)b, which translates as follows. Given $(E, \nabla) \in \mathcal{S}_{d R}(X)$, the image of the canonical morphism

$$
H^{0}(X, E) \otimes_{\mathbb{C}} \mathcal{O}_{X} \longrightarrow E
$$

is invariant under $\nabla$. Now, this follows from Remark 6.4.

Assigning to any pseudostable vector bundle $E \in \operatorname{Vect}_{0}^{\mathrm{s}}(X)$ the canonical integrable holomorphic connection on $E$ (see Theorem 6.2(2)), a functor is obtained; call it

$$
\mathbb{V}: \operatorname{Vect}_{0}^{\mathrm{s}}(X) \longrightarrow \mathcal{S}_{d R}(X) .
$$

This $\mathbb{V}$ is a tensor functor (see Remark 6.4). Hence $\mathbb{V}$ produces the section $\mathbf{v}_{X}$ to $\mathbf{u}_{X}$. Elementary group theoretic manipulations and faithful flatness of $\mathbf{j}_{X}$ [Wa, Theorem of 14.1] now tell us that $\left(\mathbf{v}_{X}, \mathbf{j}_{X}\right)$ is an isomorphism.

Note that, the natural map $\operatorname{Ext}_{\mathcal{S}_{d R}(X)}(\mathbf{1}, \mathbf{1}) \longrightarrow \operatorname{Ext}_{\mathcal{C}_{d R}(X)}(\mathbf{1}, \mathbf{1})$ is automatically an isomorphism since a vector bundle realizing an extension of $\mathcal{O}_{X}$ by itself is immediately pseudostable. It follows that the space of additive characters of $\Sigma\left(X, x_{0}\right)$ is simply $H_{d R}^{1}(X, \mathbb{C})$ (see Lemma 3.6). As the space of extensions of 1 by itself in $\operatorname{Vect}_{0}^{\mathrm{s}}(X)$ is clearly $H^{1}\left(X, \mathcal{O}_{X}\right)$, the decomposition in Theorem 6.5 recovers the decomposition $H_{d R}^{1}(X, \mathbb{C}) \simeq H^{0}\left(X, \Omega_{X}^{1}\right) \oplus H^{1}\left(X, \mathcal{O}_{X}\right)$.

Remark 6.6. Let $\widetilde{\operatorname{Vect}_{0}}(X)$ be the full subcategory of $\operatorname{Vect}_{0}^{\mathrm{s}}(X)$ defined by all holomorphic vector bundles in $\operatorname{Vect}_{0}^{\mathrm{s}}(X)$ that are direct sum of holomorphic line bundles. It is a Tannakian subcategory, and defines a quotient group scheme

$$
\pi^{\mathrm{S}}\left(X, x_{0}\right) \longrightarrow \tilde{\pi}^{\mathrm{S}}\left(X, x_{0}\right)
$$

of $\pi^{\mathrm{S}}\left(X, x_{0}\right)$. Form the proof of Theorem 6.5 it is straightforward to deduce that

$$
\Delta\left(X, x_{0}\right)=\tilde{\pi}^{\mathrm{S}}\left(X, x_{0}\right) \times \Theta\left(X, x_{0}\right),
$$

where $\Delta\left(X, x_{0}\right)$ is constructed in (5.2). 


\section{ACKNOWLEDGEMENTS}

We thank C. Simpson for a useful discussion. The first-named author is partially supported by a J. C. Bose Fellowship, and school of mathematics, TIFR, is supported by 12-R\&D-TFR-5.01-0500.

\section{REFERENCES}

[ACGH] E. Arbarello, M. Cornalba, P. A. Griffiths and J. Harris, Geometry of algebraic curves. Vol. I, Grundlehren der Mathematischen Wissenschaften, 267, Springer-Verlag, New York, 1985.

[At] M. F. Atiyah, Complex analytic connections in fibre bundles, Trans. Amer. Math. Soc. 85 (1957), 181-207.

[BD] I. Biswas and S. Dumitrescu, Riemann-Hilbert correspondence for differential systems over Riemann surfaces, arxiv.org/abs/2002.05927.

$[\mathrm{BDH}]$ I. Biswas, S. Dumitrescu and S. Heller, Irreducible flat $\mathrm{SL}(2, \mathbb{R})$-connections on the trivial holomorphic bundle, arxiv.org/abs/2003.06997.

[BHdS] I. Biswas, P. H. Hai and J. P. dos Santos, On the fundamental group schemes of certain quotient varieties. Preprint. arXiv:1809.06755v2 [math.AG].

[BG] I. Biswas and T. L. Gómez, Connections and Higgs fields on a principal bundle, Ann. Glob. Anal. Geom. 33 (2008), 19-46.

[BS] I. Biswas and S. Subramanian, Flat holomorphic connections on principal bundles over a projective manifold, Trans. Amer. Math. Soc. 356 (2004), 3995-4018.

[Bo] N. Bourbaki, Algebra. I. Chapters 1-3, Elements of Mathematics (Berlin), Springer-Verlag, Berlin, 1989.

[CDHL] G. Calsamiglia, B. Deroin, V. Heu and F. Loray, The Riemann-Hilbert mapping for $s l(2)$-systems over genus two curves, Bull. Soc. Math. Fr. 147 (2019), 159-195.

[Co] K. Corlette, Flat G-bundles with canonical metrics, Jour. Differential Geom. 28 (1988), 361382 .

[DMOS] P. Deligne, J. S. Milne, A. Ogus and K.-y. Shih, Hodge cycles, motives, and Shimura varieties, Lecture Notes in Mathematics, 900, Springer-Verlag, Berlin-New York, 1982.

[EHS] H. Esnault, P. H. Hai and X. Sun, On Nori's fundamental group scheme, Geometry and dynamics of groups and spaces, 377-398, Progr. Math., 265, Birkhäuser, Basel, 2008.

[Fr] P. Freyd, Abelian categories. An introduction to the theory of functors, Harper's Series in Modern Mathematics Harper \& Row, Publishers, New York 1964.

[GH] P. Griffiths and J. Harris, Principles of algebraic geometry. Reprint of the 1978 original. Wiley Classics Library. John Wiley \& Sons, Inc., New York, 1994.

[Ho] G. Hochschild, Basic theory of algebraic groups and Lie algebras, Graduate Texts in Mathematics, 75. Springer-Verlag, New York-Berlin, 1981.

$[\mathrm{HM}] \quad$ A. T. Huckleberry and G. A. Margulis, Invariant analytic hypersurfaces, Invent. Math. 71 (1983), 235-240.

[LT] M. Lübke and A. Teleman, The Kobayashi-Hitchin correspondence, World Scientific Publishing Co., Inc., River Edge, NJ, 1995.

[Ka] N. M. Katz, An overview of Deligne's work on Hilbert's twenty-first problem, Mathematical developments arising from Hilbert problems (Proc. Sympos. Pure Math., Vol. XXVIII, Northern Illinois Univ., De Kalb, Ill., 1974), pp. 537-557. Amer. Math. Soc., Providence, R. I., 1976.

[Ko] S. Kobayashi, Differential geometry of complex vector bundles, Publications of the Mathematical Society of Japan, 15. Kanô Memorial Lectures, 5, Princeton University Press, Princeton, NJ; Princeton University Press, Princeton, NJ, 1987.

[No] M. V. Nori, The fundamental group-scheme, Proc. Ind. Acad. Sci. (Math. Sci.) 91 (1982), $73-122$.

[Sa] N. Saavedra Rivano, Catégories Tannakiennes, Lecture Notes in Mathematics, Vol. 265, Springer-Verlag, Berlin-New York, 1972. 
[Se] C. S. Seshadri, Space of Unitary Vector Bundles on a Compact Riemann Surface, Ann. of Math. 85 (1967), 303-336.

[Si1] C. T. Simpson, Constructing variations of Hodge structure using Yang-Mills theory and applications to uniformization, Jour. Amer. Math. Soc. 1 (1988), 867-918.

[Si2] C. T. Simpson, Higgs bundles and local systems, Inst. Hautes Études Sci. Publ. Math. 75 (1992), $5-95$.

[UY] K. Uhlenbeck and S.-T. Yau, On the existence of Hermitian-Yang-Mills connections in stable vector bundles, Comm. Pure Appl. Math. 39 (1986), 257-293.

[Wa] W. C. Waterhouse, Introduction to affine group schemes, Graduate Texts in Mathematics, 66, Springer-Verlag, New York-Berlin, 1979.

[SC] Séminaire Henri Cartan, 13th year: 1960/61. Familles d'espaces complexes et fondements de la géométrie analytique. Fasc. 1 et 2: Exp. 1-21. 2nd corrected edition, École Normale Sup. Secrétariat mathématique, Paris 1962.

School of Mathematics, Tata Institute of Fundamental Research, Homi Bhabha Road, Mumbai 400005, INDiA

Email address: indranil@math.tifr.res.in

Institut de Mathématiques de Jussieu - Paris Rive Gauche, 4 Place Jussieu, Case 247, 75252 Paris Cedex 5, France

Email address: joao_pedro.dos_santos@yahoo.com

Université Côte D'Azur, CNRS, LJAD, France

Email address: dumitres@unice.fr

Mathematisches Institut, Ruprecht-Karls-Universität HeidelberG, Im Neuenheimer Feld 205, 69120 Heidelberg, Germany

Email address: seb.heller@gmail.com 